\title{
The 2004 Las Campanas/Lowell Observatory campaign II. Surface properties of Hayabusa target Asteroid 25143 Itokawa inferred from Hapke modeling
}

\author{
S. M. Lederer ${ }^{1}$, D. L. Domingue ${ }^{2}$, J. E. Thomas-Osip ${ }^{3}$, F. Vilas ${ }^{4 *}$, D. J. Osip ${ }^{5}$, S. L. Leeds ${ }^{6}$, and K. S. Jarvis ${ }^{7}$ \\ ${ }^{1}$ Dept. of Physics, CSUSB, 5500 University Parkway, San Bernardino, CA 92407 \\ ${ }^{2}$ Johns Hopkins University/Applied Physics Laboratory, 11100 Johns Hopkins Rd, Laurel, MD 20723 \\ ${ }^{3}$ The Observatories of the Carnegie Institute of Washington, Las Campanas Observatory, \\ Colina El Pino, Casilla 601, La Serena, Chile \\ ${ }^{4}$ Planetary Astronomy Group, Astromaterials Research and Exploration Science, \\ NASA Johnson Space Center/KR, Houston, TX 77058 \\ ${ }^{5}$ The Observatories of the Carnegie Institute of Washington, Las Campanas Observatory, \\ Colina El Pino, Casilla 601, La Serena, Chile \\ ${ }^{6}$ Dept. of Physics, CSUSB, 5500 University Parkway, San Bernardino, CA 92407 \\ ${ }^{7}$ ESC Group/ Hamilton Sundstrand, Houston, TX 77058
}

(Received December 8, 2006; Revised August 22, 2007; Accepted August 28, 2007; Online published February 12, 2008)

\begin{abstract}
We present an analysis of Hapke photometric modeling applied to uniform ground-based UBVRIJHK broadband data of asteroid 25143 Itokawa collected over a wide range of solar phase angles $\left(4^{\circ}-130^{\circ}\right)$ during the 2004 apparition (Thomas-Osip et al., this issue, hereafter Paper I). Our photometric analyses indicate that Itokawa has a blocky surface with properties different from other, albeit larger, S-class asteroids studied using similar Hapke modeling analyses. Images from the Hayabusa spacecraft affirm the Hapke modeling results, demonstrating the ability of Hapke photometric modeling to predict a rocky asteroid surface correctly; this is the first time that a predicted rocky surface has been observed by a spacecraft. The single particle scattering functions are dominantly forward scattering, suggesting the surface material is composed primarily of clear particles whose scattering is dictated by the particle's surface as opposed to internal scatterers (more typical of S-class asteroids), while the opposition parameters indicate that the regolith may be more compact than most of the limited number of asteroids visited by spacecraft to date. The roughness properties, single particle scattering properties, and opposition surge characteristics are all indicative of a surface where multiple scattering does not play a major role in defining the photometric properties of the regolith.
\end{abstract}

Key words: Surfaces, asteroids, photometry, regoliths, composition.

\section{Introduction}

The Japanese spacecraft Hayabusa encountered and gathered data of its near-Earth asteroid target, Itokawa, from September until early December, 2005. This mission was designed, in part, to touch down twice on its surface to collect samples of the asteroid and return them to the Earth. Hayabusa conducted two excursions to the surface of Itokawa in November, 2005 in an attempt to obtain surface samples. The first potential sample return of surface material from an asteroid, comprising these samples, is expected in 2010.

In an effort to understand the physical characteristics of this asteroid, and thereby also aid the Hayabusa mission, we obtained an extensive broadband UBVRIJHK filter dataset of Itokawa in 2004, specifically designed to provide optimal corrected solar phase curves for robust Hapke modeling of the surface scattering properties (cf. Thomas-Osip et

\footnotetext{
*Now at MMT Observatory, PO Box 210065, University of Arizona, Tucson, AZ 85721.

Copyright (c) The Society of Geomagnetism and Earth, Planetary and Space Sciences (SGEPSS); The Seismological Society of Japan; The Volcanological Society of Japan; The Geodetic Society of Japan; The Japanese Society for Planetary Sciences; TERRAPUB.
}

$a l$. , this issue, hereafter Paper I). For the first time since asteroid Itokawa was discovered and chosen for this mission, the 2004 apparition afforded us an opportunity to observe this target at low, intermediate, and high phase angles. An excellent southern hemisphere apparition during long winter nights ensured that we observed the asteroid through a full phase of its $12.132 \pm 0.0005$ hour sidereal light curve (Kaasalainen et al., 2003), essential for eliminating variations in brightness due to rotation and allowing us to accurately estimate the surface properties.

The resulting observations were modeled using Hapke's equations in an attempt to characterize the surface properties of Itokawa. Comparisons with similar modeling analyses of other asteroids provides insight into how such properties as porosity versus grain size distribution, surface roughness, albedo, and single particle scattering properties varies among asteroids.

A brief description of the observational dataset follows in Section 2. Preparation of the solar phase curves and their subsequent Hapke modeling are presented in Section 3. Finally, Section 4 provides a discussion of the surface properties and mineralogy in light of the Hapke model results and broadband colors. 


\section{Observations}

The details of the Las Campanas/Lowell Observatories Itokawa observing campaign for the 2004 apparition are provided in Paper I. In summary, we obtained complete UBVRIJHK $(0.36,0.44,0.54,0.63,0.85,1.25,1.65$, and 2.15 microns, respectively) rotational light-curve coverage of Itokawa with solar phase angle coverage varying by filter. Solar phase curves for BVRI observations cover the largest range from 4-129 degrees, allowing investigations of both the backscattering and forward-scattering properties of the surface regolith, The near infrared solar phase curves (JHK) and additional visible $U$ band phase curves cover an intermediate range of solar phase angles from 11-49 and 11-69 degrees, respectively. To supplement the data from the 2004 apparition, a subset of data from the 2001 apparition (Lederer et al., 2005) with sufficient temporal sampling were included; the underlying rotational light-curve from the solar phase curve was re-fit for these data, using the same procedures as in Paper I, and removed. These 2001 apparition data overlap and extend the intermediate region of the solar phase curves for BVRI from 40-90 degrees (cf. figure 16, Paper I).

\section{Hapke Modeling Analysis}

The Hapke equations (Hapke, 1981, 1984, 1986, 2002) are a set of bi-directional reflectance equations derived from radiative transfer and a set of physical assumptions that can be used to model photometric observations. The parameters within the Hapke equations can be correlated to surface properties and used to compare regolith properties from one solar system object to another. The following subsections describe the processing of the Itokawa observations into a photometric data set that can be modeled using a subset of the Hapke equations in an attempt to discern Itokawa's regolith properties, especially in comparison to other asteroids that have been similarly modeled and visited by spacecraft.

\subsection{Solar phase curves}

Thomas-Osip et al. (Paper I) derived solar phase curves in units of absolute magnitude for eight colors (U-K) using the 2004 apparition observations. These solar phase curves were corrected for rotational phase curve variations and represent brightness variations as a function of solar phase angle (Sun-asteroid-Earth) only. The same methodology for removing rotational phase variations and converting to absolute magnitudes was applied to a subset of observations from the 2001 apparition taken by Lederer et al. (2005). The reprocessing of the Lederer et al. (2005) observations gave smaller residual variations in the calculated solar phase curves (than those found in the earlier paper adopting a different correction method) and consistent results in the overlap region between the two apparitions.

Absolute magnitude units were converted into absolute reflectance values using the relationship $I / F=10^{-0.4 m_{a}}$, where $m_{a}$ is the absolute magnitude and $I / F$ is the absolute reflectance. Figure 1 shows an example solar phase curve, in this case for the V filter data. Some variation remains due to asteroid shape uncertainties that are unaccounted for in the methodology used for converting to units of absolute magnitude. A daily median value was calculated for each

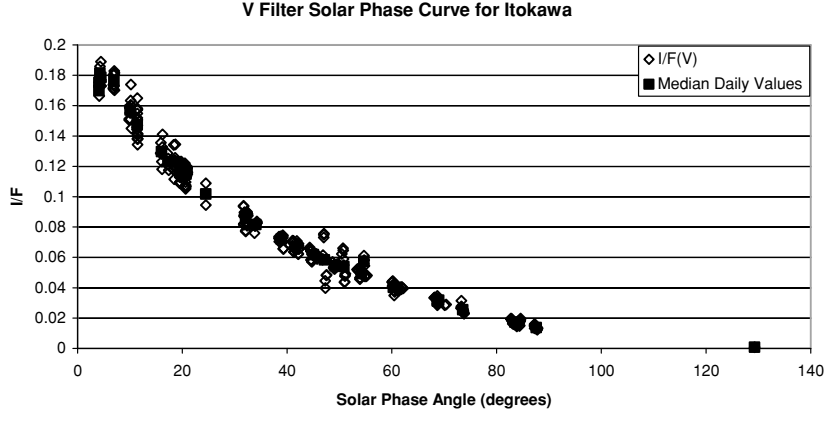

Fig. 1. Itokawa $\mathrm{V}$ filter observations are shown. The diamonds are the complete data set after rotational phase variations have been removed and converted to absolute reflectance. The squares show the median daily values, which were used to derived the Hapke model parameters.

day's set of rotationally corrected observations, producing a solar phase curve describing the general, average surface of the asteroid Itokawa.

\subsection{Hapke photometric modeling}

The resulting solar phase curves were modeled using Hapke's photometric equations (Hapke, 1981, 1984, 1986) for each filter. An updated set of photometric equations, which incorporate additional parameters to describe the opposition surge due to coherent backscatter (Hapke, 2002), was not chosen for this analysis since the data set is not extensive enough in the opposition regime (solar phase angle $=0-5^{\circ}$ ) to support the updated model. While the Hapke equations selected for this analysis incorporate many assumptions and simplifications, they have been shown to accurately estimate planetary photometric behavior to within 10\% (Cheng and Domingue, 2000), which is within the error bars of the solar phase curves being modeled in this study. The set of photometric equations chosen for this study have also been used to model other asteroid data sets, providing context for our results.

The Hapke equation for the absolute reflectance, $I / F$, is given as

$$
\begin{aligned}
\frac{I}{F}= & {\left[\left\{\frac{w}{8}[(1+B(\alpha)) P(\alpha)-1]+\frac{r_{o}}{2}\left(1-r_{o}\right)\right\}\right.} \\
& \cdot\left\{1-\sin \left(\frac{\alpha}{2}\right) \tan \left(\frac{\alpha}{2}\right) \ln \left[\cot \left(\frac{\alpha}{4}\right)\right]\right\} \\
& \left.+\frac{2}{3} r_{o}\left(\frac{\sin (\alpha)+(\pi-\alpha) \cos (\alpha)}{\pi}\right)\right] S(\alpha, \theta),
\end{aligned}
$$

where $w$ is the single-particle scattering albedo, $\alpha$ is the solar phase angle, and $\theta$ is the surface roughness parameter. The term $r_{o}$ is given by

$$
r_{o}=\frac{(1-\sqrt{1-w})}{(1+\sqrt{1-w})},
$$

and the opposition effect term, $B(\alpha)$, is given by the approximation (Hapke, 1986)

$$
B(\alpha)=\frac{B_{o}}{\left[1+\frac{\tan (\alpha / 2)}{h}\right]},
$$

where $B_{o}$ describes the amplitude of the opposition effect and $h$ describes the width of the opposition peak. This ap- 
proximation has been demonstrated to describe the opposition effect in terms of shadow-hiding mechanisms (Hapke, 1986). The single-particle scattering function, $P(\alpha)$, used in the Hapke equation was a double Henyey-Greenstein function of the form

$$
P(\alpha)=\frac{(1-c)\left(1-b^{2}\right)}{\left[1-2 b \cos \alpha+b^{2}\right]^{\frac{3}{2}}}+\frac{c\left(1-b^{2}\right)}{\left[1+2 b \cos \alpha+b^{2}\right]^{\frac{3}{2}}},
$$

where $b$ describes the amplitude of the scattering lobes and $c$ describes the relative scattering between the forward (high $c$ values) and backward (low $c$ values) scattering directions.

The last term in Eq. (1), $S(\alpha, \theta)$, is the correction for surface roughness where the surface roughness parameter, $\theta$, is defined as the average slope over the resolution element of the detector (Hapke, 1984). Shkuratov et al. (2005) have shown through Monte Carlo ray-tracing methods that interpretations of surface roughness based on the Hapke roughness parameter need to be made with care. Multiple scattering effects on bright objects produces an underestimation of roughness by $\theta$, and in some of their simulations $\theta$ did not accurately predict the surface roughness where large-scale roughness was present. They also demonstrated that albedo effects on photometric behavior can be interchanged with surface roughness effects. Interpretations of $\theta$ should be made in context with multiple scattering and albedo properties.

3.2.1 Modeling methods The observed solar phase curves for each filter were modeled using a least squares grid search over the Hapke parameter space using the same algorithm from Domingue et al. (2002) and Lederer et al. (2005). The least squares grid search routine searches over the Hapke parameters $w, B_{o}, h, b, c$, and $\theta$, described above. The routine finds the set of Hapke parameters which minimizes $\chi$ and can vary all parameters simultaneously. The delimiter, $\chi$, is defined as

$$
\chi=\frac{\left([I / F]_{\text {observed }}-[I / F]_{\text {measured }}\right)^{2}}{N},
$$

where $[I / F]_{\text {observed }}$ is the observed reflectance, $[I / F]_{\text {measured }}$ is the disk-integrated brightness calculated from the Hapke equations, and $N$ is the number of data points or observations comprising the solar phase curve. The modeling results, where all Hapke parameter values were allowed to vary, are provided in Tables 1 through 8.

3.2.2 Error analysis The error bars for each of the Hapke parameters is, at minimum, no smaller than the grid size of the least-squares grid search. The smallest grid size used on these data sets was 0.01 for the Hapke parameters $w, B_{o}, h, b$, and $c$. The smallest grid size for the surface roughness parameter, $\theta$, was $5^{\circ}$.

Each of the parameters within the Hapke equations are best constrained with measurements within certain ranges of solar phase angle. The single particle scattering parameters $(b$ and $c$ ), for example, require observations in both the backward scattering $\left(0^{\circ}\right.$ to $70^{\circ}$ solar phase $)$ and forward scattering $\left(110^{\circ}\right.$ to $180^{\circ}$ solar phase $)$ regions. The opposition region $\left(0^{\circ}\right.$ to $5^{\circ}$ solar phase $)$ constrains the opposition parameters $\left(B_{o}\right.$ and $\left.h\right)$. The parameter $B_{o}$ can affect the shape of the model solar phase curve from $0^{\circ}$ to $20^{\circ}$ (Harris et al., 1989), thus measurements in the opposition region are needed to separate $B_{o}$ value effects from those of other parameters. It has been noted that variations in the shape of the disk-integrated solar phase curves produced by different values of the single particle scattering function can be compensated for by changes in the surface roughness parameter, which in turn can be compensated for by variations in the single scattering albedo (Harris et al., 1989; Shkuratov et al., 2005). Observations at very high solar phase angle (> $120^{\circ}$ ) can begin to constrain and distinguish between the effects these parameters have on the shape of the solar phase curve. Disk-resolved measurements (which were not available at the time of this study) can further constrain this subset of the Hapke model parameters to produce more unique solutions.

The B, V, R, I solar phase curves had observations ranging from $4^{\circ}$ to $129^{\circ}$, where as the $\mathrm{J}, \mathrm{H}, \mathrm{K}$, observations ranged between $11^{\circ}$ and $49^{\circ}$, and the $\mathrm{U}$ filter observations ranged from $11^{\circ}$ to $69^{\circ}$. The backward scattering region $\left(0^{\circ}\right.$ to $70^{\circ}$ solar phase $)$ is reasonably constrained within all the data sets, however the forward scattering region $\left(110^{\circ}\right.$ to $180^{\circ}$ solar phase) is partially constrained only within the $\mathrm{B}, \mathrm{V}, \mathrm{R}$, and I data sets. This will result in larger uncertainties in the single particle scattering function parameters for the $\mathrm{U}, \mathrm{J}, \mathrm{H}$, and $\mathrm{K}$ data sets. The resulting error bars for the parameters are listed in Tables 1 to 8 . These error bars are derived from modeling results using different initial assumptions based on data coverage. This is done to decouple the different parameter effects for the same region of the solar phase curve. For example, the U, J, H, K filter data do not have coverage in the opposition region, thus solutions were found based on different assumptions for the opposition effect parameters (ranging from null values to those values obtained by Domingue et al. (2002) for Eros). Ranges for the other parameter values were found that resulted in models within the error bars of the measurements, and thus provided the error estimates for these parameters.

3.2.3 Modeling results Tables 1 to 8 list the solution one parameter values for each filter modeled with the Hapke equations. Figures 2 to 9 show the Hapke equation fits (labeled as "Hapke model solution") compared to the observations (labeled as "Median Daily Values").

\subsection{Photometric characteristics}

3.3.1 Single particle characteristics Comparison of the single particle scattering albedo with the reflectance spectrum taken of Itokawa on March 23, 2001 (Abell et al., submitted), and a reflectance spectrum taken March 6, 2001 (Binzel et al., 2001) are shown in Fig. 10. The single particle scattering albedo displays the same trend with wavelength as the spectral observations. This correlation between single particle scattering albedo and spectral observations was also seen for Eros (Clark et al., 2002b) and confirms that the Hapke model results are consistent with the compositional results obtained via spectrophotometry. In the V-band, where the single particle scattering albedo has been commonly reported for other asteroids, the single particle scattering albedo for Itokawa is significantly higher than that seen for an average S-class asteroid (Domingue et al., 2002). Verbiscer and Veverka (1995) translated the 
Table 1. U filter Hapke parameter values.

\begin{tabular}{ccc}
\hline Parameter & Value & Error \\
\hline$w$ & 0.53 & \pm 0.07 \\
$B_{o}$ & 1 & N/A \\
$h$ & 0.05 & N/A \\
$b$ & 0.53 & \pm 0.1 \\
$c$ & 0.89 & \pm 0.1 \\
$\theta$ & $40^{\circ}$ & $\pm 10^{\circ}$ \\
$\chi$ & $8.08 \mathrm{E}-06$ & \\
\hline
\end{tabular}

N/A: value is unconstrained by the data set.

Table 2. B filter Hapke parameter values.

\begin{tabular}{ccc}
\hline Parameter & Value & Error \\
\hline$w$ & 0.66 & \pm 0.04 \\
$B_{o}$ & 0.04 & \pm 0.1 \\
$h$ & 0.25 & \pm 0.1 \\
$b$ & 0.61 & \pm 0.04 \\
$c$ & 0.89 & \pm 0.04 \\
$\theta$ & $40^{\circ}$ & $\pm 5^{\circ}$ \\
$\chi$ & $7.04 \mathrm{E}-05$ & \\
\hline
\end{tabular}

Table 3. V filter Hapke parameter values.

\begin{tabular}{ccc}
\hline Parameter & Value & Error \\
\hline$w$ & 0.7 & \pm 0.04 \\
$B_{o}$ & 0.02 & \pm 0.1 \\
$h$ & 0.141 & \pm 0.1 \\
$b$ & 0.59 & \pm 0.04 \\
$c$ & 0.87 & \pm 0.04 \\
$\theta$ & $40^{\circ}$ & $\pm 5^{\circ}$ \\
$\chi$ & $7.21 \mathrm{E}-05$ & \\
\hline
\end{tabular}

Table 4. R filter Hapke parameter values.

\begin{tabular}{ccc}
\hline Parameter & Value & Error \\
\hline$w$ & 0.71 & \pm 0.04 \\
$B_{o}$ & 0.05 & \pm 0.1 \\
$h$ & 0.231 & \pm 0.1 \\
$b$ & 0.56 & \pm 0.04 \\
$c$ & 0.84 & \pm 0.04 \\
$\theta$ & $40^{\circ}$ & $\pm 5^{\circ}$ \\
$\chi$ & $9.17 \mathrm{E}-05$ & \\
\hline
\end{tabular}

IAU two-parameter $(H-G)$ photometric descriptions, based on the Lumme-Bowell photometric model, into equivalent Hapke parameter values. The $H-G$ magnitude system is comprised of the absolute visual magnitude, $H$, and a slope parameter, $G$, to characterize the asteroid phase curve. The conversion, discussed by Thomas-Osip et al. (Paper I) to Hapke parameter space, would place the value for Itokawa in the range seen for E-, R-, and V-class asteroids.

Figure 11 shows a plot of the single particle scattering functions corresponding to the Hapke parameter values listed in Tables 1 through 8 . All of the single particle scattering functions are forward scattering, however the $\mathrm{J}, \mathrm{H}$, and $\mathrm{K}$ functions are less so with backscattering playing a stronger role in the scattering behavior. The $\mathrm{B}, \mathrm{V}, \mathrm{R}$, and I observations cover solar phase angles between $4^{\circ}$ and $129^{\circ}$
Table 5. I filter Hapke parameter values.

\begin{tabular}{ccc}
\hline Parameter & Value & Error \\
\hline$w$ & 0.73 & \pm 0.04 \\
$B_{o}$ & 0.23 & \pm 0.1 \\
$h$ & 0.46 & \pm 0.1 \\
$b$ & 0.59 & \pm 0.04 \\
$c$ & 0.89 & \pm 0.04 \\
$\theta$ & $40^{\circ}$ & $\pm 5^{\circ}$ \\
$\chi$ & 0.000132 & \\
\hline
\end{tabular}

Table 6. H filter Hapke parameter values.

\begin{tabular}{ccc}
\hline Parameter & Value & Error \\
\hline$w$ & 0.69 & \pm 0.2 \\
$B_{o}$ & 0.90 & N/A \\
$h$ & 0.12 & N/A \\
$b$ & 0.41 & \pm 0.35 \\
$c$ & 0.67 & \pm 0.35 \\
$\theta$ & $40^{\circ}$ & N/A \\
$\chi$ & $5.62 \mathrm{E}-05$ & \\
\hline
\end{tabular}

N/A: value is unconstrained by the data set.

Table 7. J filter Hapke parameter values.

\begin{tabular}{ccc}
\hline Parameter & Value & Error \\
\hline$w$ & 0.61 & \pm 0.2 \\
$B_{o}$ & 0.95 & N/A \\
$h$ & 0.42 & N/A \\
$b$ & 0.43 & \pm 0.35 \\
$c$ & 0.78 & \pm 0.35 \\
$\theta$ & $40^{\circ}$ & N/A \\
$\chi$ & $5.95 \mathrm{E}-06$ & \\
\hline
\end{tabular}

N/A: value is unconstrained by the data set.

Table 8. K filter Hapke parameter values.

\begin{tabular}{ccc}
\hline Parameter & Value & Error \\
\hline$w$ & 0.58 & \pm 0.2 \\
$B_{o}$ & 0.97 & N/A \\
$h$ & 0.111 & N/A \\
$b$ & 0.31 & \pm 0.35 \\
$c$ & 0.44 & \pm 0.35 \\
$\theta$ & $40^{\circ}$ & N/A \\
$\chi$ & $9.02 \mathrm{E}-06$ & \\
\hline
\end{tabular}

N/A: value is unconstrained by the data set.

degrees, which better constrains the single particle scattering behavior. In contrast, the $U$ filter only covers phase angles to $69^{\circ}$ and the $\mathrm{J}, \mathrm{H}$, and $\mathrm{K}$ filters only to $49^{\circ}$ degrees. These data sets do not adequately sample the forward scattering regime $\left(>110^{\circ}\right)$, thus the $\mathrm{B}, \mathrm{V}, \mathrm{R}$, and I curves better describe the particle scattering behavior. Comparisons of similar single particle scattering curves provided in Domingue et al. (2002) for Eros, Gaspra, Ida, and average S-class asteroids, at wavelengths approximating the $\mathrm{V}$ filter, show that the particles within the regolith of these objects are dominantly backward scattering, with only a minor to non-existent forward scattering component.

In Fig. 11, one can see the general trend for the single par- 
U filter Solar Phase Curve for Itokawa

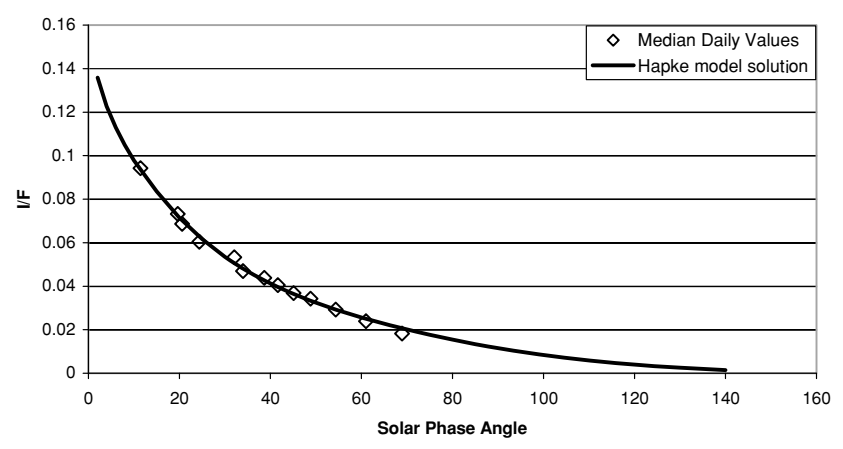

Fig. 2. This is a plot of the Itokawa $U$ filter observations reduced to median daily values (diamonds) versus the Hapke model solution to the solar phase curve (solid line).

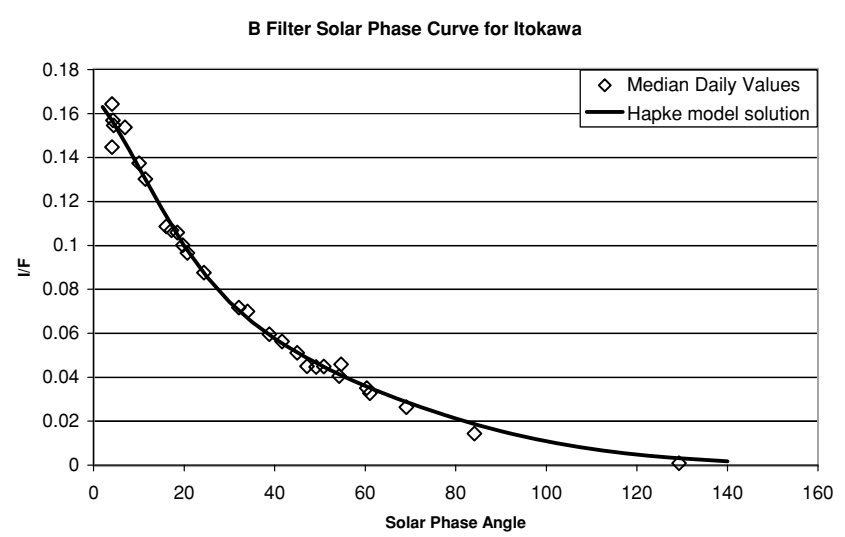

Fig. 3. As in Fig. 2, for the Itokawa B filter observations.

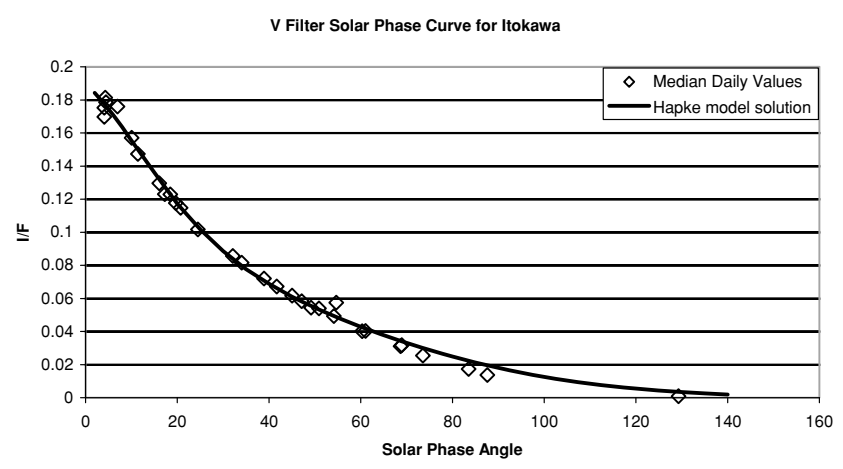

Fig. 4. As in Fig. 2, for the Itokawa V filter observations.

ticle scattering functions to be more backscattering at longer wavelengths. However, the Henyey-Greenstein parameters, $c$ and $b$, show little to no variation with wavelength within their errors, thus this trend with wavelength should be considered a subtle, if existent, trend. The solutions to the single particle scattering function, because of the limits within the observational data, should be interpreted as a demonstration of the possible range in scattering behavior of the regolith particles.

McGuire and Hapke (1995) and Hartman and Domingue (1998) have mapped single particle scattering function parameters to a subset of particle characteristic types. Their results are shown in Fig. 12 along with the positions of the

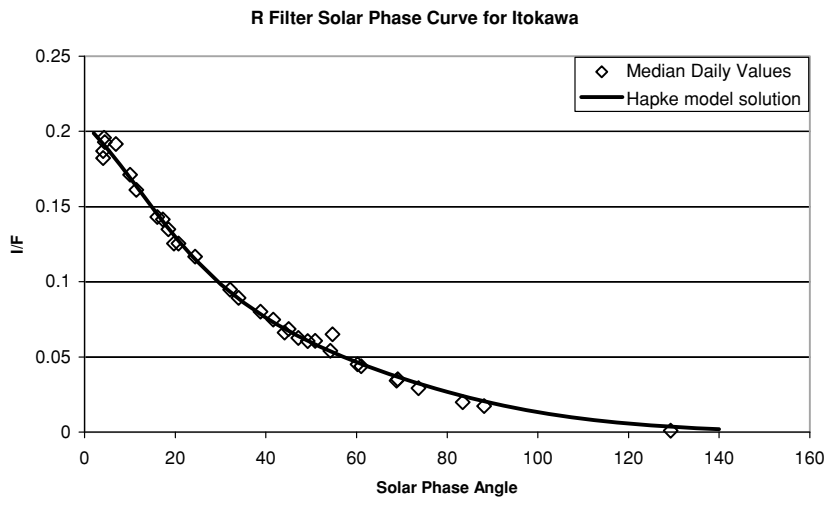

Fig. 5. As in Fig. 2, for the Itokawa R filter observations.

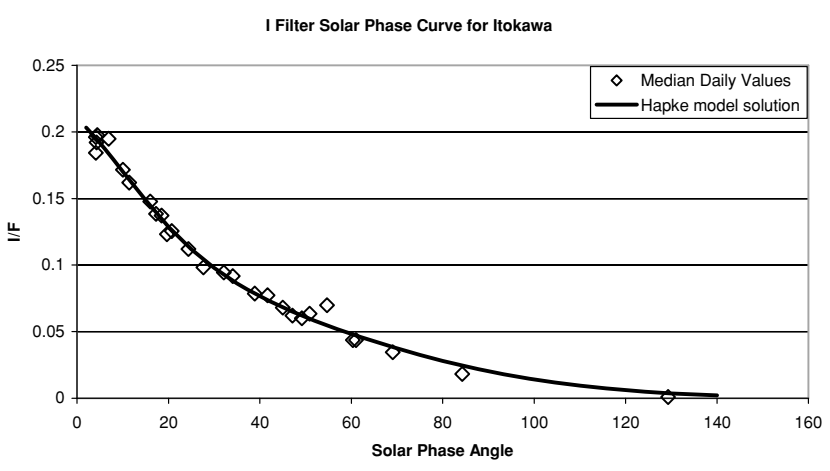

Fig. 6. As in Fig. 2, for the Itokawa I filter observations.

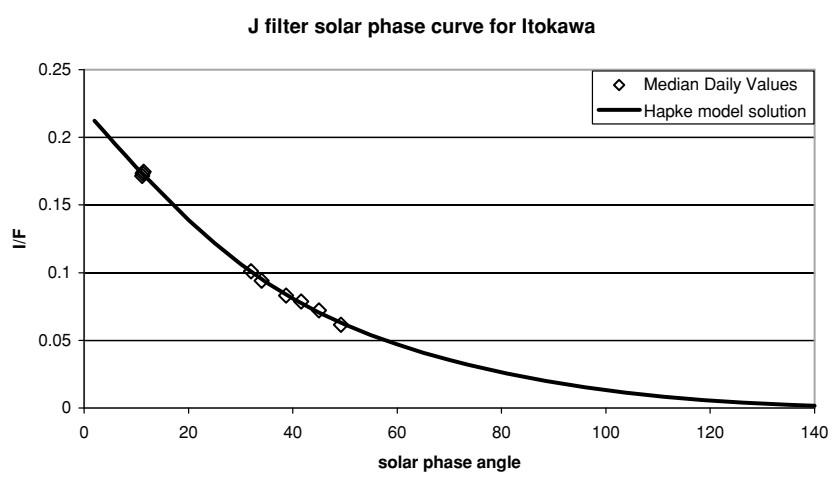

Fig. 7. As in Fig. 2, for the Itokawa J filter observations.

Itokawa modeling results superimposed on the map. For comparison, positions of Eros, Gaspra, Ida, Dactyl, and the Moon (based on modeling results of $\sim \mathrm{V}$ wavelength observations from Helfenstein and Veverka (1987), Helfenstein et al. (1994, 1996), Domingue et al. (2002)) are also superimposed on the map. These results indicate that the scattering behavior in the majority of the filters plots in those regions described by clear particles where any scattering centers are associated with the particle's surface. Only the parameter values in the $\mathrm{K}$ filter map to a region indicative of internal scatterers for that wavelength. In contrast, the modeling results for the other asteroids (and Moon) plot in regions characterized by particles with moderate to high amounts of internal scatterers. This indicates that Itokawa has a regolith with properties atypical of asteroids observed to date by spacecraft. 
H filter solar phase curve for Itokawa

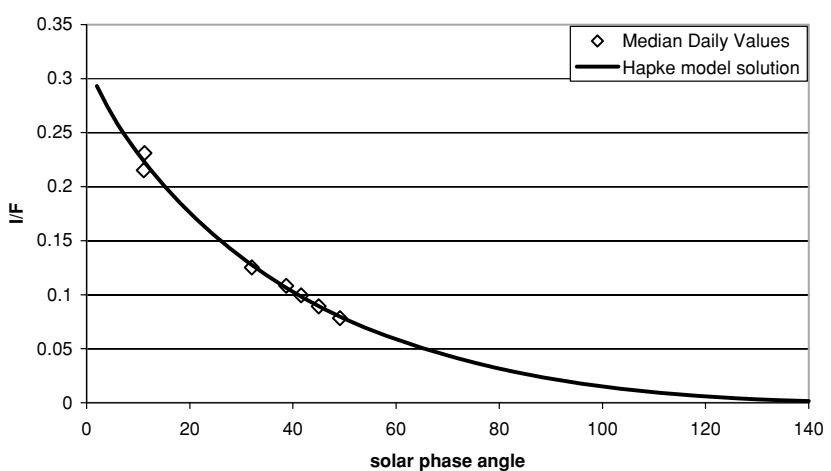

Fig. 8. As in Fig. 2, for the Itokawa $\mathrm{H}$ filter observations.

K filter solar phase curve for Itokawa

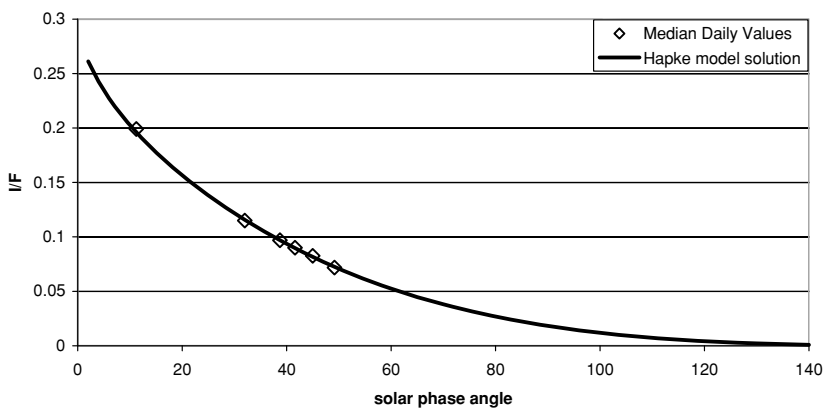

Fig. 9. As in Fig. 2, for the Itokawa K filter observations.

Single Scattering Albedo

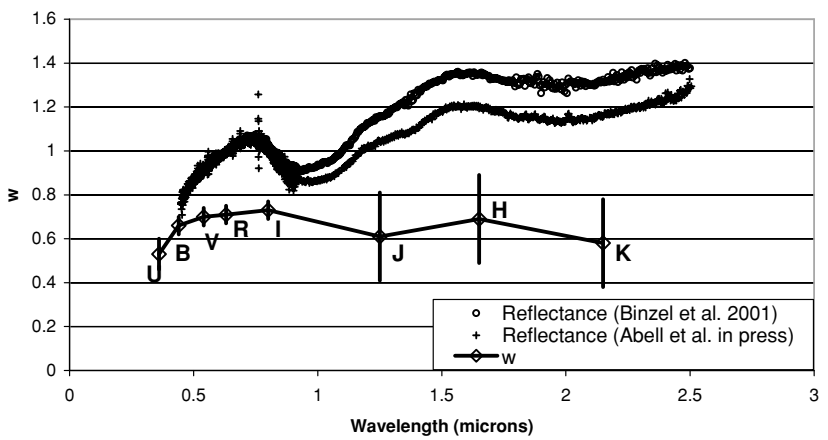

Fig. 10. Reflectance spectra of Itokawa (Abell et al., in press; Binzel et al., 2001) are compared to the variation in the single scattering albedo, $w$, as a function of wavelength. The single scattering albedo follows the spectral trend with wavelength.

3.3.2 Opposition characteristics The filters containing opposition information (B, V, R, I), meaning observations down to $4^{\circ}$ solar phase angle, have low values for the opposition amplitude (ranging from 0.02 to 0.23 ) and broad values for the opposition surge width (ranging from 0.141 to 0.46$)$. This is in sharp contrast to the large amplitude and narrow width values measured for average, albeit larger, $\mathrm{S}$ class asteroids (as seen in the $\mathrm{V}$ filter).

The opposition amplitude shows no trend with wavelength, while the opposition surge width, $h$, generally increases with wavelength. This is an opposite trend in comparison to that seen for Eros (Clark et al., 2002b). The opposition effect in planetary regoliths is produced by a com-
Single Particle Scattering Function

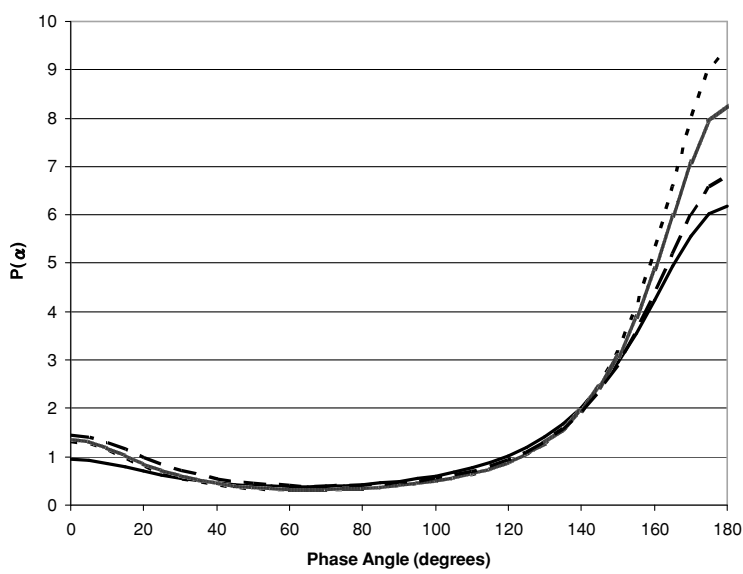

Single Particle Scattering Function

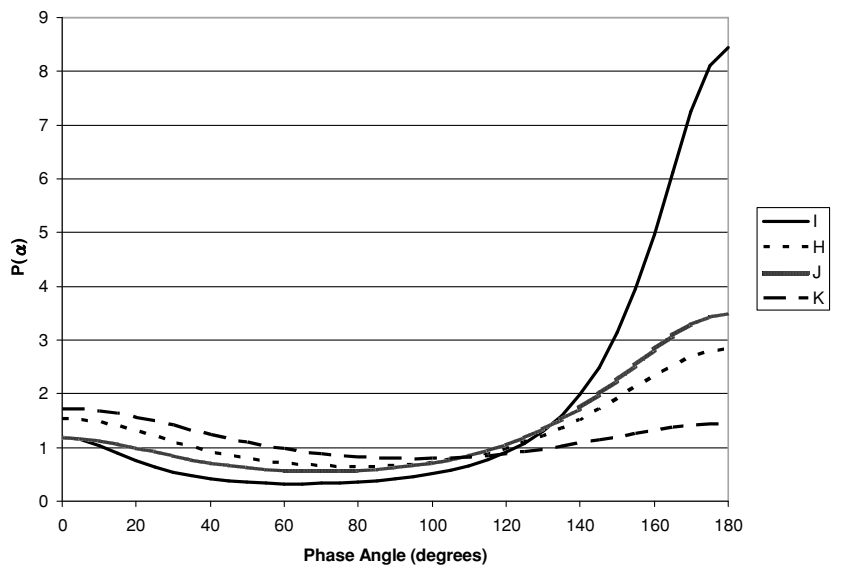

Fig. 11. These plots shows the predicted single particle scattering function from the Hapke modeling results to the various filter observations of Itokawa. All the scattering functions have a strong forward scattering component. (A) The $\mathrm{U}$ filter results are plotted by a solid line, the B filter results are plotted by a short dashed line, the $\mathrm{V}$ filter results are plotted by a chain-link line, the $\mathrm{R}$ filter results are plotted by a long dashed line. (B) The I filter results are plotted by a solid line, the $\mathrm{H}$ filter results are plotted by a short dashed line, the $\mathrm{J}$ filter results are plotted by a chain-link line, and the $\mathrm{K}$ filter results are plotted by a long dashed line.

bination of two mechanisms: shadow-hiding (Hapke, 1986) and coherent backscatter (Hapke, 2002). The shadowhiding mechanism can produce a broader opposition effect that is independent of wavelength. The coherent backscatter mechanism is thought to produce a narrower opposition surge than that produced by shadow-hiding and does have a wavelength dependence (Hapke et al., 1998). Thus the fact that we see a relatively wide and low opposition would argue that the shadow-hiding mechanism is in play here. The wavelength dependence indicates that coherent backscatter may play a role in producing the opposition surge measured on Itokawa, but the coherently backscattered surge is most likely narrower than we could have detected with a minimum phase angle of $4^{\circ}$. Due to the broad nature of the opposition surge measured by the observations, we proceed with an analysis of the opposition effect parameters assuming the dominate formation mechanism is shadow-hiding. 


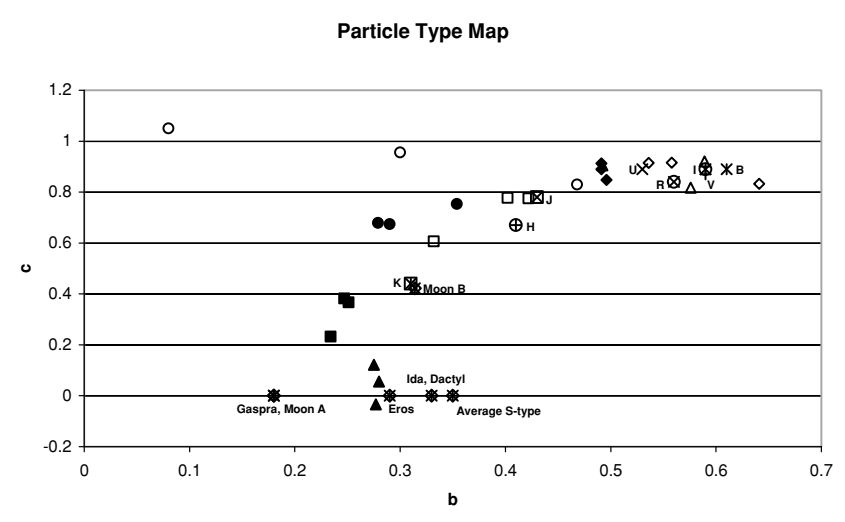

Fig. 12. This diagram shows the regions within the Henyey-Greenstein single particle scattering function parameter space that correspond to the model particle types examined by McGuire and Hapke (1995) and Hartman and Domingue (1998). Open diamonds outline the region characterized by smooth, clear, spherical particles; open squares outline the region characterized by clear, rough surfaced, spherical particles; open triangles outline the region characterized by clear, pitted surface, spherical particles; open circles outline the region characterized by clear, irregularly shaped particles; closed diamonds outline the region characterized by spherical particles with a low amount of internal scatterers; closed squares outline the region characterized by spherical particles with a moderate amount of internal scatterers; closed triangles outline the region characterized by spherical particles with a high amount of internal scatterers; and closed circles outline the region characterized by agglutinate particles. A more detailed description of the particle types is provided in McGuire and Hapke (1995). The derived values from the Hapke modeling of the Itokawa telescopic observations are also plotted, and labeled for comparison (U filter is shown by a cross, B filter is shown by an asterisk, $\mathrm{V}$ filter is shown by a plus, $\mathrm{R}$ filter is shown by a circled cross, I filter is shown by a circled asterisk, $\mathrm{H}$ filter is shown by a circled plus, $\mathrm{J}$ filter is shown by a squared cross, and $\mathrm{K}$ filter is shown by a squared asterisk). Also shown for comparison are several asteroids and the Moon, labeled and denoted by a diamond-asterisk.

This allows us to compare with past analyses of other asteroid regoliths.

The Hapke opposition width parameter, $h$, has been related to porosity and grain size distribution by the relationship

$$
h=-\left(\frac{3}{8}\right) Y \ln (\rho),
$$

where $\rho$ is the porosity and $Y$ is the particle size distribution (Hapke, 1986). Particle size distributions have been derived for the Moon; however the applicability of such distributions to small asteroids, such as Itokawa, must be made with a clear understanding of the assumptions applied. The following analyses have been applied to many other small asteroids, and provide a preliminary point for comparison of regolith variations between small objects.

The lunar regolith is derived from impact processes, where the gravity well of the Moon is large enough to retain the fine-grained portion of the impact ejecta, and over time the regolith has been ground-down to contain a significant fine-grained portion. For small asteroids, such as Itokawa, the processes for producing the regolith are not well understood. A regolith on a small asteroid can be a result of the accretionary process (thus producing what is termed an "accretional regolith") or a result of impact processes, where the grains are much coarser than that observed for the Moon (Clark et al., 2001). One reason for the coarser grain size

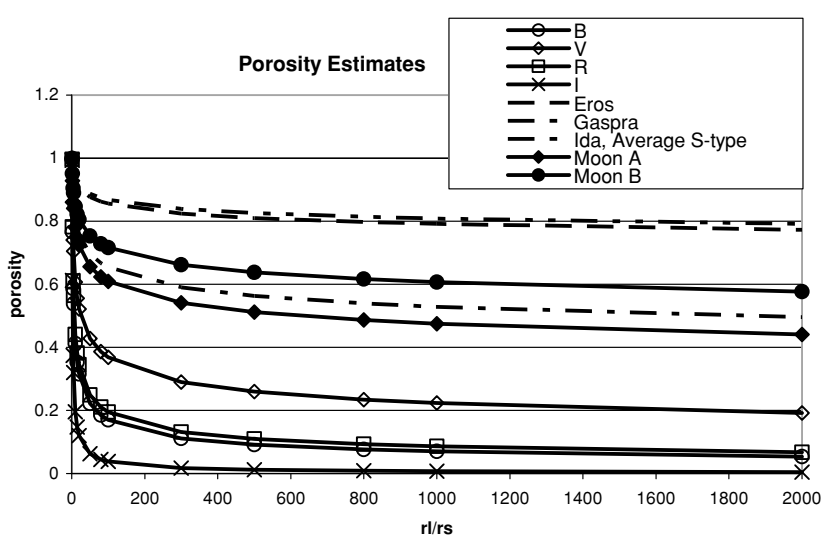

Fig. 13. This plot shows porosity estimates based on the Hapke model opposition parameter values for the B (line with circles), V (line with diamonds), R (line with squares), and I (line with crosses) Itokawa observations. For comparison, similar porosity estimates are shown for Eros (long dashed line), Gaspra (dash-dot line), Ida (dash-dot-dot line), and the Moon (line with closed diamonds are based on Helfenstein and Veverka (1987) results, line with closed circles are based on Hartman and Domingue (1998) results). Similar porosity estimates based on model parameters for average S-class asteroids plot identical to Ida. The porosity estimates assume a lunar-like particle size distribution, which may not be representative of the regolith of small asteroids. Note that the estimates for Itokawa are much lower than those of the other objects shown.

is that it requires a much more energetic impact to produce fine-grained material, which will then have more energy and be more readily lost to space.

Estimates on porosity based on a lunar-like particle distribution, to first order, should remove a fine-grained component. The particle size distribution function, $Y$, for the Moon has been given by

$$
Y=\frac{\sqrt{3}}{\ln \left(r_{l} / r_{s}\right)},
$$

where $r_{l}$ and $r_{s}$ are the radii of the largest and smallest particles, respectively. The ratio of $r_{l}$ to $r_{s}$ will be smaller for Itokawa than for the Moon due to the removal of the finegrained component. Figure 13 plots the range of porosity values for various particle size ratios based on the Hapke model solutions to the Itokawa $\mathrm{B}, \mathrm{V}, \mathrm{R}$, and I observations. For comparison, similar porosity values based on $\sim \mathrm{V}$ wavelength observations are plotted for asteroids Eros, Gaspra, Ida, Average S-class, and the Moon. These comparisons show that the regolith of Itokawa is more compact than on these other objects. This is consistent with the Hayabusa AMICA images and ground-based thermal inertia findings that indicate a gravel-to-brecciated rock type of regolith (Müller et al., 2005; Yano et al., 2006). For example, evidence suggests a largely rocky or pebbly surface: the smallest-sized particles are roughly $3 \mathrm{~mm}$ diameter in Muses Sea, the smoothest area of the asteroid corresponding to a geopotential low on the surface. In addition, there is effectively no evidence for cratering on the surface, as would be expected of a regolithic surface, suggesting that the surface is not coherent enough to hold the shape of an impact crater. Boulders much larger than those expected to form as crater ejecta following an impact event are found on the surface. Collectively, these support the conclusion 
Table 9. Filter dependent geometric albedo, Bond albedo, and phase integral.

\begin{tabular}{cccc}
\hline Filter & Geometric albedo & Bond albedo & Phase integral \\
\hline U & $0.15 \pm 0.05$ & $0.012 \pm 0.01$ & $0.08 \pm 0.02$ \\
B & $0.17 \pm 0.02$ & $0.0153 \pm 0.01$ & $0.09 \pm 0.01$ \\
V & $0.19 \pm 0.02$ & $0.0209 \pm 0.01$ & $0.11 \pm 0.01$ \\
R & $0.21 \pm 0.02$ & $0.0252 \pm 0.01$ & $0.12 \pm 0.01$ \\
I & $0.21 \pm 0.02$ & $0.0252 \pm 0.01$ & $0.12 \pm 0.01$ \\
J & $0.22 \pm 0.12$ & $0.0264 \pm 0.04$ & $0.12 \pm 0.01$ \\
H & $0.32 \pm 0.12$ & $0.048 \pm 0.03$ & $0.15 \pm 0.01$ \\
K & $0.28 \pm 0.12$ & $0.0364 \pm 0.04$ & $0.13 \pm 0.01$ \\
\hline
\end{tabular}

that the asteroid formed from the joining of two bodies that probably resulted from the gravitational re-accretion of scattered material that formed from a major impact into a larger parent asteroid.

3.3.3 Surface roughness The value for the surface roughness parameter $\left(40^{\circ}\right)$ is independent of wavelength as is expected from the Hapke model assumptions. All fits return a high value, similar to what is found in disk-integrated analyses of Eros (Domingue et al., 2002). This is commensurate with the irregular shape and blocky nature of the surface, as seen in images returned from Hayabusa (Saito et al., 2006). A roughness value commensurate with surface topography, a forward scattering single particle scattering function, and a shallow broad opposition effect are all indicative of a surface regolith where multiple scattering does not play a major role.

3.3.4 Geometric and Bond albedos, phase integral Table 9 lists the filter dependent values for the geometric albedo, Bond albedo, and phase integral for Itokawa. The geometric albedo, $A_{g}$, is defined as the disk-integrated reflectance of a surface at $\alpha=0^{\circ}$ relative to a same-size Lambert disk under the same observing geometry. The Bond or spherical albedo is defined as the fraction of incident light scattered in all directions by the surface. The Bond albedo is given by $q A_{g}$, where $q$ is the phase integral. The phase integral is given by

$$
q=2 \int_{0}^{\pi} \frac{\phi(\alpha)}{\phi\left(\alpha=0^{\circ}\right)} \sin (\alpha) d \alpha,
$$

where $\phi(\alpha)$ is defined in Eq. (1) as $I / F$. The value for the phase integral was found using the Romberg method of integration. The errors for the albedos and phase integral values in each filter are derived by calculating the values for each solution set. The maximum difference between the values for a given filter was used as the error bar, while the actual values are those based on the Hapke model solution parameter sets given here.

Table 10 compares equivalent $\mathrm{V}$ filter geometric albedo, Bond albedo, and phase integral for several asteroids. While the geometric albedo for Itokawa is commensurate with the values for the other asteroids listed, the values for the Bond albedo and phase integral are consistently low, further exemplifying the atypical nature of the regolith for Itokawa.

\subsection{Comparisons with previous studies}

Not surprisingly, given the much wider phase angle coverage combined with the much more complete rotational-
Table 10. Equivalent V-filter geometric albedo, Bond albedo, and phase integral values.

\begin{tabular}{cccc}
\hline Object & Geometric albedo & Bond albedo & Phase integral \\
\hline Itokawa & $0.19 \pm 0.02$ & $0.0209 \pm 0.01$ & $0.11 \pm 0.01$ \\
Eros $^{\mathrm{a}}$ & $0.290 \pm 0.02$ & $0.12 \pm 0.02$ & $0.39 \pm 0.02$ \\
Gaspra $^{\mathrm{b}}$ & $0.23 \pm 0.06$ & $0.12 \pm 0.03$ & $0.47 \pm 0.04$ \\
Ida $^{\mathrm{c}}$ & $0.206 \pm 0.032$ & $0.081 \pm 0.017$ & $0.34 \pm 0.02$ \\
Dactyl $^{\mathrm{c}}$ & $0.198 \pm 0.05$ & $0.073 \pm 0.021$ & $0.32 \pm 0.03$ \\
Average S-class $^{\mathrm{c}, \mathrm{d}}$ & 0.22 & 0.084 & 0.33 \\
\hline
\end{tabular}

${ }^{\mathrm{a}}$ Domingue et al. (2002). ${ }^{\mathrm{b}}$ Helfenstein et al. (1994). ${ }^{\mathrm{c}} \mathrm{Helfenstein}$ et al. (1996). ${ }^{\mathrm{d}}$ Helfenstein and Veverka (1989).

phase coverage offered in the present study (including opposition, forward scattering and backscattering regions) compared to that available for the 2001 apparition, our best fit Hapke model solutions are not consistent with those found in Lederer et al. (2005). However, we find that applying the updated rotational correction methodology to the available subset of data from 2001 as incorporated in the present study indicates complete consistency at intermediate solar phase angles where the data overlap between the two apparitions. Furthermore, the model fit of the synodic light-curve variation and subsequent removal from the 2001 apparition data results in substantially less scatter in the derived solar phase curve. This kind of complete rotational analysis was not possible with the limited 2001 dataset as the rotational period of this asteroid was not known prior to the 2001 apparition; 2001 was the first apparition after this asteroid was discovered (in 1998) and chosen as the spacecraft target, thus little data existed up to that point. Our 2004 campaign required a five-week observing run to fully cover the rotational period of $12.132 \pm 0.0005$ hours (Kaasalainen et al., 2003), which was derived from 2001 data and used to plan the 2004 campaign. Clearly, the most productive modeling of surface properties derived from groundbased observations benefits strongly from access to a uniform dataset with sufficient temporal sampling to accurately model rotation - and-sufficient solar phase coverage to include the three distinct regions of opposition, forward scattering and backscattering.

\subsection{Conclusions from Hapke modeling}

Images of the rocky Itokawa surface from the Hayabusa mission (c.f., Fujiwara et al., 2006) coupled with the results of the Hapke modeling presented here demonstrate for the first time that Hapke modeling of ground-based photometry is correctly predictive of surface roughness. The Hapke analysis here suggests that Itokawa has a rocky or coarsenot fine, particulate-surface, unlike the Moon. The geometric albedo of 0.23 calculated under the assumption of a rocky surface is consistent with the geometric albedo of 0.22 for an average S-class asteroid (Helfenstein and Veverka, 1989). As discussed above, AMICA images from the Hayabusa spacecraft show that the surface of Itokawa consists of a rocky or pebbly surface, having pebbles with diameters ranging from $3 \mathrm{~mm}$ to tens of meters. We conclude that Hapke modeling of Visible to Near-IR photometry of asteroids can be utilized effectively to characterize asteroid surface properties in support, for example, of future spacecraft missions or NEA characterization efforts. 


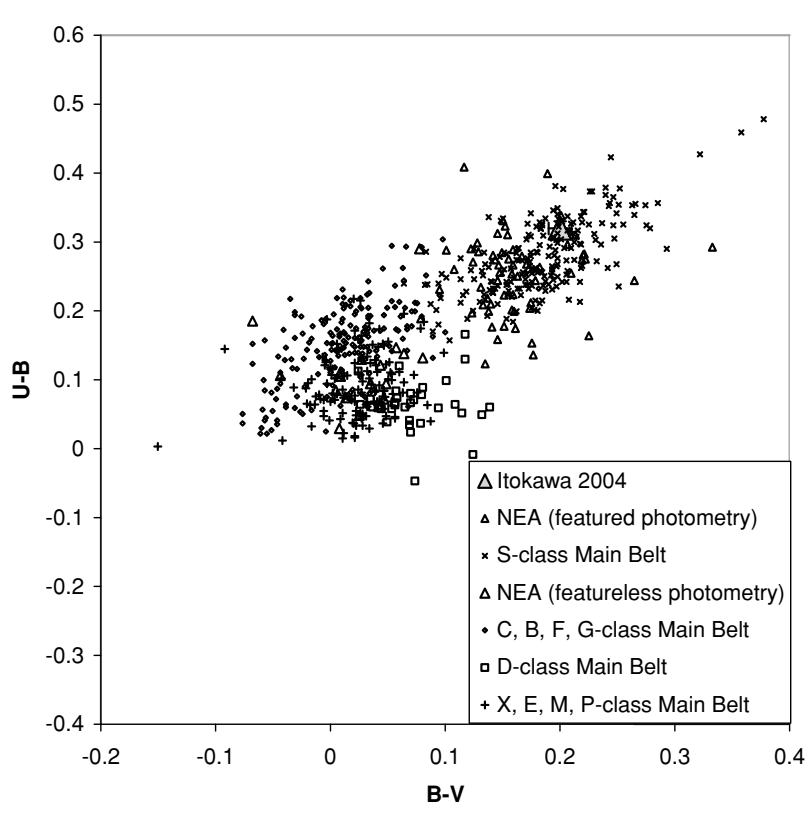

Fig. 14. The U-B color generally represents a measure of the presence and strength of the $\mathrm{Fe}^{3+} \mathrm{UV} /$ blue Intervalence Charge Transfer Transition (IVCT) absorption. U-B colors for Itokawa indicate a strong IVCT absorption, suggesting it is an S-class asteroid.

\section{Surface Mineralogy}

Images of the rocky Itokawa surface from the Hayabusa mission (c.f., Fujiwara et al., 2006) coupled with the results of the Hapke modeling presented here demonstrate for the first time that Hapke modeling of ground-based photometry is correctly predictive of surface roughness. The Hapke analysis here suggests that Itokawa has a rocky or coarsenot fine, particulate-surface, unlike the Moon. The geometric albedo of 0.23 calculated under the assumption of a rocky surface is consistent with the geometric albedo of 0.22 for an average S-class asteroid (Helfenstein and Veverka, 1989).

As concluded in Paper I, the geometric albedo and colors derived from this uniform dataset suggest that Itokawa appears to be an average main-belt S-class asteroid. Figures 14-16 provide color-color plots highlighting Itokawa among the various main-belt asteroid classes as well as resolved and unresolved near-Earth asteroid photometry. In addition, colors reported by Lederer et al. (2005) for the 2001 apparition are included although both the number and quality of the color observations from the 2004 apparition (in particular U-B) supplant these earlier results. The difference in U-B results in the 2001 apparition versus 2004 is not surprising; the 2001 apparition included only $3 \mathrm{U}$ observations (plotted separately in Lederer et al. (2005)) while the 2004 data includes over $125 \mathrm{U}$ observations in the average color. Figure 14 shows that the strength of the $\mathrm{Fe}^{3+} \mathrm{UV} /$ blue intervalence charge transfer transition as represented by the U-B vs. B-V color-color plot is consistent with the average S-class asteroid. The B-V vs. V-R colors (Fig. 15) also suggest a moderately strong UV/blue absorption. The V-I vs. V-R plot in Fig. 16 shows that the presence and strength of the mafic silicate absorption band near $1.0 \mu \mathrm{m}$ is also consistent with the average $\mathrm{S}$-class asteroids.

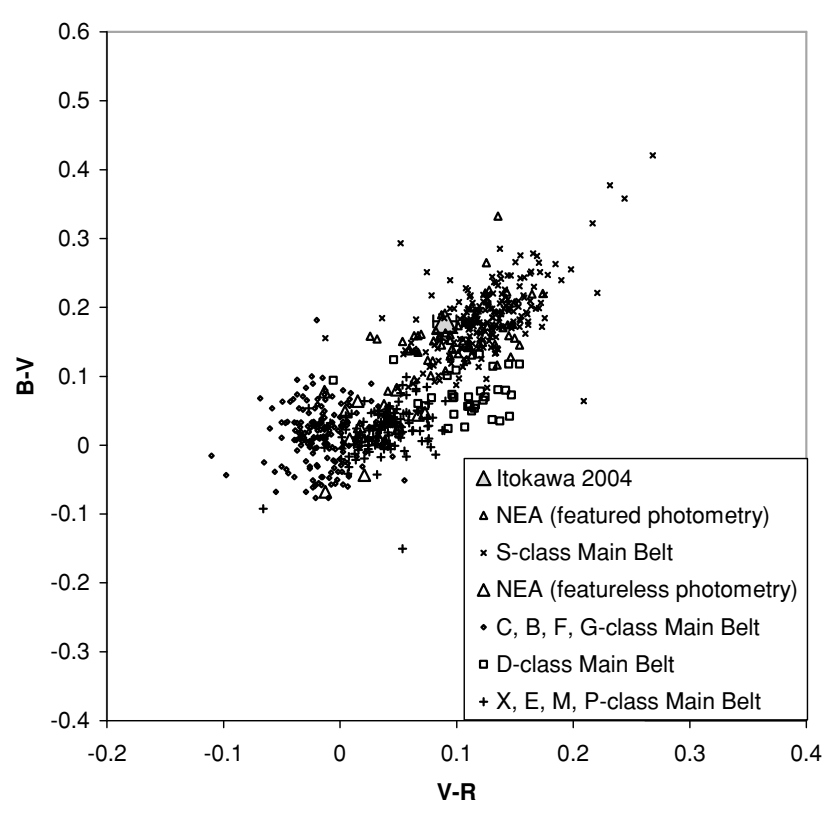

Fig. 15. B-V vs. V-R colors suggest that Itokawa is somewhat of an outlier to the other S-class asteroids. The B-V colors also suggest a strong $\mathrm{UV} /$ blue absorption, consistent with the U-B colors.

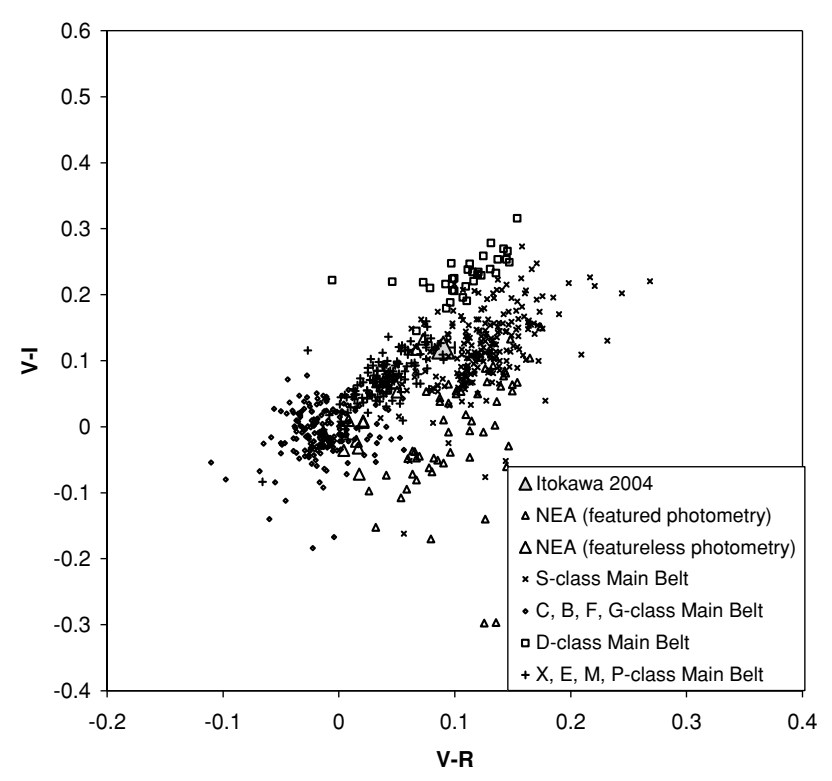

Fig. 16. V-I vs. V-R broadband colors show the presence of a mafic silicate absorption feature, again suggesting Itokawa is an S-class asteroid.

Conventional theory about "space weathering" suggests that S-class asteroids are comprised of mafic silicate minerals similar to those minerals found in ordinary chondrites, and a thin layer of surface material altered by some form of space weathering to produce the redder reflectance spectra with shallower absorption features observed today (c.f., Clark et al., 2002a; Chapman, 1996; Pieters et al., 2000). Near-Earth asteroids probably represent a population of objects recently moved to their current, unstable orbits predominantly (though not exclusively) by an impact that fractured a larger parent body. The smaller daughter bodies we observe today might be expected to have different spectra since they were not affected by space weathering processes. 
Their spectral properties should form a continuum between the properties of S-class asteroids and properties of ordinary chondrite meteorites (Rabinowitz, 1998; Binzel et al., 2001). Reflectance spectra of the Q-class asteroids seen in the NEA population demonstrate the spectral transition from ordinary chondrites to S-class asteroids. The spectra of the smaller NEA asteroids should appear much less weathered, therefore much less like the main-belt S-class asteroid spectra.

Itokawa is a surprise, in this case, as it appears to have the spectral and albedo properties of a presumably spaceweathered and larger S-class asteroid, although its dimensions are considerably smaller. Its surface appears rocky and, therefore, less subjected to micrometeoroid impact that could create a particulate surface. It does not fit the above profile of the smaller NEA that has experienced much less space weathering and alteration than the larger main-belt S-class asteroids.

Two possibilities have been proposed that would explain this apparent conundrum. First, an alternate theory has also been proposed to address the NEA population. Here, both $\mathrm{S}$-class and Q-class asteroids exist in roughly equal proportions at different diameters among the NEAs, and no transition between the S-class spectral properties and the ordinary chondrite meteorites needs to be invoked (Whiteley, 2001). The ordinary chondrites are likely derived from the Q-class asteroids (Whiteley, 2001; McFadden et al., 1984). Smaller Q-class asteroids have been identified among the inner main-belt objects. In this case, Itokawa represents a case of a smaller S-class asteroid, not weathered and having large surface roughness.

Second, laboratory laser irradiation of rocky ordinary chondrite meteorites by Sasaki et al. (2006) show darkening and reddening of their surfaces, although to a lesser degree than for particulate meteorite samples. Based on this example, Sasaki et al. (2006) suggest that rocky surfaces of asteroids can also be darkened and reddened without necessarily forming a particulate regolith. They thus propose that the surface of asteroid Itokawa could have been darkened and reddened through space weathering processes, and that there is no conflict between the rocky, rougher surface and the spectral reflectance characteristics of Itokawa (Sasaki et al., 2006).

Finally, although the JHK photometry lacks sufficient spectral resolution for detailed mineralogical analyses, the solar-corrected relative reflectance JHK values show even less increase in reflectance with increased wavelength (reddening) than the spectra of Abell et al. (in press), already less reddened than the spectrum from Binzel et al. (2001) (Fig. 10). While the broadband characteristics of the JHK filters could cause the filter values to be slightly inconsistent with the narrowband spectrophotometry centered at the same wavelengths, the photometry is clearly less reddened. The reduced reddening supports the interpretation that conventional space weathering did not affect the surface of Itokawa.

\section{Conclusions}

Results of Hapke photometric modeling for 25143 Itokawa, a small asteroid that looks very different from any other asteroid visited by a spacecraft, indicate that its physical surface properties also differ from typical S-class asteroids, although its color photometry is consistent with average, albeit larger, S-class asteroids. All fits for the surface roughness parameter yield a high value, indicative of a blocky surface, which was confirmed by Hayabusa spacecraft images.

Unlike other S-class asteroids studied in-situ by spacecraft (e.g. Eros, Gaspra, Ida), the single particle scattering functions of Itokawa at different wavelengths are dominantly forward scattering. These results suggest that Itokawa's regolith is composed of clear particles whose scattering is due primarily to the particle's surface. In contrast, the average S-class asteroid's surface is typically comprised of particles with high levels of internal scattering. Itokawa's opposition characteristics suggest that the regolith is more compact than expected, however, we note that our opposition data (ideally $0-5^{\circ}$ ) is limited (minimum phase angle of $4^{\circ}$ ). While the geometric albedo is consistent with other S-class asteroids studied to date, the Bond albedo and phase integrals differ from the typical, larger S-class asteroids. These differences are consistent with rocky surface material. However, the color values spanning the UV to the near-IR are, unexpectedly, consistent with main-belt, Sclass asteroids. It is possible that Itokawa is representative of smaller asteroids. Further studies of small asteroids are required.

These ground-based and space-based observations and modeling of Itokawa demonstrate that Hapke modeling can be used to predict correctly the surface mechanical properties of asteroids. This has implications beyond the characterization of the properties of Itokawa: Hapke modeling of the surface properties of asteroids remains a cost-effective and energy-efficient means of assessing asteroid properties. The uses for this process are many and varied: Future spacecraft missions can use this knowledge to design sample return missions. Scientists have a tool with which they can characterize the mechanical properties of potentially hazardous asteroids. Therefore, this stands as one result of the Hayabusa mission that can directly contribute to the future scientific exploration of asteroids.

Acknowledgments. We wish to thank Y. G. Shkuratov and an anonymous referee for helpful comments that have improved this manuscript. This work was supported in part by the NASA MUCERPI program (Lederer) and the NASA/Hayabusa program (Vilas). This work was also supported in part by the NASA Planetary Astronomy Program. Deborah Domingue acknowledges support from the NEAR Data Analysis Program (NAG512646). Special thanks to the support staff at Lowell and Las Campanas Observatories. IRAF is distributed by the National Optical Astronomy Observatories, which is operated by the Association of Universities for Research in Astronomy, Inc. (AURA) under cooperative agreement with the National Science Foundation.

\section{References}

Abell, P. A., F. Vilas, K. S. Jarvis, M. J. Gaffey, and M. S. Kelley, Mineralogical Composition of (25143) Itokawa 1998 SF36 from visible and Near-Infrared Reflectance Spectroscopy: Evidence for Partial Melting, Meteoritics Planet. Sci., (in press).

Binzel, R. P., A. S. Rivkin, S. J. Bus, J. M. Sunshine, and T. H. Burbine, MUSES-C target asteroid (25143) Itokawa: A reddened ordinary chondrite, Meteoritics Planet. Sci., 36, 1167-1172, 2001. 
Chapman, C. R., S-Type Asteroids, Ordinary Chondrites, and Space Weathering: The Evidence from Galileo's Fly-bys of Gaspra and Ida, Meteoritics Planet. Sci., 31, 699-725, 1996.

Cheng, A. F. and D. L. Domingue, Radiative transfer models for light scattering from planetary surfaces, J. Geophys. Res., 105, 9477-9482, 2000.

Clark, B. E., P. Lucey, P. Helfenstein, J. F. Bell III, C. Peterson, J. Veverka, T. McConnochie, M. S. Robinson, B. Bussey, S. L. Murchie, N. I. Izenberg, and C. R. Chapman, Space weathering on Eros: Constraints from albedo and spectral measurements of Psyche crater, Meteoritics Planet. Sci., 36, 1617-1637, 2001.

Clark, B. E., B. Hapke, C. Pieters, and D. Britt, Asteroid Space Weathering and Regolith Evolution, in Asteroids III, edited by B. Bottke et al., 585599, Univ. of Arizona Press, Tucson, 2002a.

Clark, B. E., P. Helfenstein, J. F. Bell, C. Peterson, J. Veverka, N. I. Izenberg, D. Domingue, D. Wellnitz, and L. McFadden, NEAR Infrared Spectrometer Photometry of Asteroid 433 Eros, Icarus, 155, 189-204, 2002b.

Domingue, D. L., M. Robinson, B. Carcich, J. Josesph, P. Thomas, and B. E. Clark, Disk integrated photometry of 433 Eros, Icarus, 155, 205-219, 2002.

Fujiwara, A., J. Kawaguchi, D. K. Yeomans, M. Abe, T. Mukai, T. Okada, J. Saito, H. Yano, M. Yoshikawa, D. J. Scheeres, O. Barnouin-Jha, A. F. Cheng, H. Demura, R. W. Gaskell, N. Hirata, H. Ikeda, T. Kominato, H. Miyamoto, A. M. Nakamura, R. Nakamura, S. Sasaki, and K. Uesugi, The Rubble-Pile Asteroid Itokawa as Observed by Hayabusa, Science, 312, 1330-1334, 2006.

Hapke, B., Bidirectional reflectance spectroscopy. I. Theory., J. Geophys. Res., 86, 3039-3054, 1981.

Hapke, B., Bidirectional reflectance spectroscopy. III. Correction for macroscopic roughness, Icarus, 59, 41-59, 1984.

Hapke, B., Bidirectional reflectance spectroscopy. IV. The extinction coefficient and the opposition effect, Icarus, 67, 264-280, 1986.

Hapke, B., Bidirectional reflectance spectroscopy. 5. The coherent backscatter opposition effect and anisotropic scattering, Icarus, 157, 523-534, 2002.

Hapke, B., R. Nelson, and W. Smythe, The Opposition Effect of the Moon: Coherent Backscatter and Shadow Hiding, Icarus, 133, 89-97, 1998.

Harris, A. W., J. W. Young, L. Contreiras, T. Dockweiler, L. Belkora, H. Salo, W. D. Harris, E. Bowell, M. Poutanen, R. P. Binzel, D. J. Tholen, and S. Wang, Phase relations of high albedo asteroids-The unusual opposition brightening of 44 NYSA and 64 Angelina, Icarus, 81, 365374, 1989.

Hartman, B. and D. Domingue, Scattering of light by individual particles and the implications for models of planetary surfaces, Icarus, 131, 421448, 1998.

Helfenstein, P. and J. Veverka, Photometric properties of lunar terrains derived from Hapke's equation, Icarus, 72, 342-357, 1987.

Helfenstein, P. and J. Veverka, Physical characterization of asteroid surfaces from photometric analysis, in Asteroids II, edited by R. P. Binzel, T. Gehrels, M. S. Matthews, 557-593, Univ. of Arizona Press, Tucson, 1989.

Helfenstein, P., J. Veverka, P. C. Thomas, D. P. Simonelli, P. Lee, K. Klaasen, T. V. Johnson, H. Breneman, J. W. Head, and S. Murchie, Galileo photometry of Asteroid 951 Gaspra, Icarus, 107, 37, 1994.

Helfenstein, P., J. Veverka, P. C. Thomas, D. P. Simonelli, K. Klaasen, T. V. Johnson, F. Fanale, J. Granahan, A. S. McEwen, M. Belton, and C. Chapman, Galileo photometry of Asteroid 243 Ida, Icarus, 120, 48-65, 1996.
Kaasalainen, M., T. Kwiatkowski, M. Abe, J. Piironen, T. Nakamura, Y. Ohba, B. Dermawan, T. Farnham, F. Colas, S. Lowry, P. Weissman, R. J. Whitely, D. J. Tholen, S. M. Larson, M. Yoshikawa, I. Toth, and F. P. Velichko, CCD photometry and model of MUSES-C target 25143 Itokawa, Astron. Astrophys., 405, L29-L32, 2003.

Lederer, S. M., D. L. Domingue, F. Vilas, M. Abe, T. L. Farnham, K. S. Jarvis, S. C. Lowry, Y. Ohba, P. R. Weissman, L. M. French, H. Fukai, S. Hasegawa, M. Ishiguro, S. M. Larson, and Y. Takagi, Physical characteristics of Hayabusa target Asteroid 25143 Itokawa, Icarus, 173, 153-165, 2005.

McFadden, L. A., M. J. Gaffey, and T. B. McCord, Mineralogicalpetrological characterization of near-earth asteroids, Icarus, 59, 25-40, 1984.

McGuire, A. F. and B. W. Hapke, An experimental study of light scattering by large irregular particles, Icarus, 113, 134, 1995.

Müller, T. G., T. Sekiguchi, M. Kaasalainen, M. Abe, and S. Hasegawa, Thermal infrared observations of the Hayabusa spacecraft target asteroid 25143 Itokawa, Astron. Astrophys., 443, 347-355, 2005.

Pieters, C. M., L. A. Taylor, S. K. Noble, L. P. Keller, B. Hapke, R. V. Morris, C. C. Allen, D. S. McKay, and S. Wentworth, Space weathering on airless bodies: Resolving a mystery with lunar samples, Meteorit. Planet. Sci., 35, 1101, 2000.

Rabinowitz, D. L., Size and orbit dependent trends in the reflectance colors of Earth-approaching asteroids, Icarus, 134, 342-346, 1998.

Saito, J., H. Miyamoto, R. Nakamura, M. Ishiguro, T. Michikami, A. M. Nakamura, H. Demura, S. Sasaki, N. Hirata, C. Honda, A. Yamamoto, Y. Yokota, T. Fuse, F. Yoshida, D. J. Tholen, R. W. Gaskell, T. Hashimoto, T. Kubota, Y. Higuchi, T. Nakamura, P. Smith, K. Hiraoka, T. Honda, S. Kobayashi, M. Furuya, N. Matsumoto, E. Nemoto, A. Yukishita, K. Kitazato, B. Dermawan, A. Sogame, J. Terazono, C. Shinohara, and H. Akiyama, Detailed Images of Asteroid 25143 Itokawa from Hayabusa, Science, 312, 1341-1344, 2006.

Sasaki, S., T. Nimura, T. Hiroi, M. Ishiguro, N. Hirata, M. Abe, Y. Ueda, A. Yamamoto, and B. E. Clark, Space Weathering of Rock Surface Without Regolith: Laboratory Simulation of Spectral Change, LPSC XXXVII, abstract 1705, 2006.

Shkuratov, Yu. G., D. G. Stankevich, D. V. Petrov, P. C. Pinet, Au. M. Cord, and Y. H. Daydou, Interpreting photometry of regolith-like surfaces with different topographies: shadowing and multiple scatter, Icarus, 173, 3$15,2005$.

Thomas-Osip, J. E., S. M. Lederer, D. J. Osip, F. Vilas, D. Domingue, K. Jarvis, and S. L. Leeds, The 2004 Las Campanas/Lowell Observatory Itokawa campaign: I. Simultaneous visible and near-infrared photometry of the Hayabusa mission target, Earth Planets Space, 60, this issue, 39-48, 2008.

Verbiscer, A. J. and J. Veverka, Interpretation of the IAU two-parameter magnitude system for asteroids in terms of Hapke photometric theory, Icarus, 115, 369-373, 1995.

Whiteley, R. J., A compositional and dynamical survey of the near Earth asteroids, Ph.D. thesis, Univ. Hawaii, 2001.

Yano, H., T. Kubota, H. Miyamoto, T. Okada, D. Scheeres, Y. Takagi, K. Yoshida, M. Abe, S. Abe, O. Barnouin-Jha, A. Fujiwara, S. Hasegawa, T. Hashimoto, M. Ishiguro, M. Kato, J. Kawaguchi, T. Mukai, J. Saito, S. Sasaki, and M. Yoshikawa, Science, 312, 1350-1353, 2006.

S. M. Lederer (e-mail: slederer@csusb.edu), D. L. Domingue, J. E. Thomas-Osip, F. Vilas, D. J. Osip, S. L. Leeds, and K. S. Jarvis 